\title{
Os professores apoiam a autonomia dos alunos nas aulas de educação física?
}

\author{
Luciane Cristina Arantes da Costa ${ }^{\mathrm{a}, *} \odot$, Patricia Carolina Borsato Passos ${ }^{\mathrm{a}} \odot$, Vânia de Fátima Matias de Souza ${ }^{\mathrm{a}}$, \\ Jorge Both ${ }^{\mathrm{b}} \odot$, Lenamar Fiorese ${ }^{\mathrm{a}} \odot$
}

Palavras-chave:

Educação física; Autonomia;

Clima de aprendizagem; Aluno.

\begin{abstract}
RESUMO
Este estudo analisou a percepção dos estudantes em relação ao apoio à autonomia dos professores nas aulas de educação física $(n=100)$. Foi usado o instrumento Learning Climate Questionnaire (LCQ). Para comparações entre o pré e pós-teste foi usado o teste de Wilcoxon e entre os grupos o de Kruskal-Wallis, seguido da comparação múltipla das medianas $(p \leq 0,05)$. Os resultados demonstraram que os índices de apoio à autonomia não se alteraram ao longo das aulas, principalmente no sexo feminino (grupo experimental). Conclui-se que o programa de intervenção não foi suficiente para apresentar alterações na percepção dos alunos em relação ao apoio à autonomia oferecido pelos professores.
\end{abstract}

\section{Keywords:}

Physical education; Personal autonomy; Learning climate; Students.

\section{Palavras Chave:}

Educación física; Autonomía personal; Clima de aprendizaje; Estudiantes.

\begin{abstract}
This study analyzed the students' perception regarding support for the autonomy of teachers in physical education classes $(n=100)$. We used the instrument Learning Climate Questionnaire (LCQ). For comparisons between the pre and post-test was used Wilcoxon test, and between groups the Kruskal Wallis test, followed by multiple comparisons of medians ( $p \leq 0.05)$. The results showed that the levels of support for autonomy have not changed during the lessons, especially in females (experimental group). In conclusion: the intervention program was not enough to introduce changes in the perception of students regarding support to the autonomy offered by teachers.
\end{abstract}

\section{RESUMEN}

Este estudio analizó la percepción de los estudiantes en relación con el apoyo a la autonomía por parte de los profesores en las clases de educación física $(n=100)$. Se utilizó el instrumento Learning Climate Questionnaire (LCQ). Para las comparaciones entre el pre y post-test se utilizó la prueba de Wilcoxon, y entre los grupos la prueba de Kruskal Wallis, seguida de comparaciones múltiples de las medianas $(p \leq 0,05)$. Los resultados mostraron que los niveles de apoyo para la autonomía no han cambiado durante las clases, especialmente en las mujeres (grupo experimental). En conclusión, el programa de intervención no fue suficiente para introducir cambios en la percepción de los estudiantes sobre el apoyo a la autonomía ofrecido por los profesores.

\footnotetext{
a Universidade Estadual de Maringá, Departamento de Educação Física, Programa de Pós-Graduação Associado em Educação Física, Maringá, PR, Brasil.

b Universidade Estadual de Londrina, Departamento de Educação Física, Programa de Pós-Graduação Associado em Educação Física, Londrina, PR, Brasil.
}

\footnotetext{
*Autor correspondente:

Luciane Cristina Arantes da Costa

E-mail: luarantes100@gmail.com
} 


\section{INTRODUÇÃO}

O clima de aprendizagem, também denominado de clima motivacional, parece ser um requisito fundamental para enfrentamentos necessários à melhoria da motivação, persistência, emoções e alterações no comportamento dos estudantes no contexto educacional. Ao reconhecer esses aspectos, a comunidade educacional poderia ter um dos pontos de partida para avaliar o contexto e propor mudanças nas diretrizes educacionais. Ames (1992) verificou em seus estudos que o clima de aprendizagem tem influência no estabelecimento de metas dos estudantes. Nesse sentido, em um ambiente em que o professor oferece apoio à autonomia, o clima de aprendizagem tornase adequado, tem como consequência o aumento da motivação intrínseca dos estudantes. Ao contrário, quando o clima de aprendizagem é inadequado ou controlado, os professores não fornecem respostas significativas para o processo de ensino e aprendizagem.

Estudos relacionados a essa temática demonstraram que os estudantes são mais motivados, interessados e envolvidos ativamente nas tarefas quando os professores oferecem apoio às atividades desenvolvidas, demonstram o quanto o clima de aprendizagem é fundamental no processo educativo (Deci et al., 1981).

Para Guimarães e Boruchovitch (2004), o apoio à autonomia pode ser influenciado pelas características da personalidade do professor e por habilidades adquiridas, que podem ser aprendidas ao longo dos anos de trabalho, ao conseguir se colocar no lugar do aluno, reconhecer seus sentimentos, usar linguagem não controladora e oferecer informações para tomadas de decisão.

Reeve e Jang (2006) avaliaram os comportamentos instrucionais de apoio à autonomia e controle dos professores. Para os autores, ao promover a autonomia, o professor aprende a ouvir os estudantes, favorece sua escolha, permite que façam as tarefas em seus próprios caminhos e, além disso, elogia os alunos, fornece informações adequadas durante as aulas.

No presente estudo, ao analisar a percepção dos escolares em relação ao apoio à autonomia, usou-se como apoio teórico a teoria da autodeterminação (TAD) (Deci e Ryan, 2000, 1985). No contexto educativo, a TAD tem aprofundado o conhecimento da interferência do ambiente escolar na motivação dos estudantes. Em geral, quanto maior o envolvimento nas tarefas, mais motivado intrinsecamente o indivíduo se apresenta, é o lócus de causalidade interno em que as razões do envolvimento na atividade ocorrem em função de seu interesse e satisfação (Deci e Ryan, 2000; Ryan e Connell, 1989).
Cox et al. (2008) verificaram que ao apoiar a autonomia houve um aumento da motivação autodeterminada e melhoria no clima motivacional, melhorou-se a aprendizagem dos estudantes. Por outro lado, em estudo feito por Assor et al. (2005), verificouse que os professores controladores despertaram ansiedade no comportamento dos estudantes.

Assim, embora as investigações tenham analisado a motivação dos estudantes nas aulas de educação física, existe uma lacuna na literatura no que se refere à percepção dos alunos em relação ao apoio à autonomia na execução de um programa de intervenção pedagógica. Nesse sentido, o presente estudo objetivou verificar a percepção dos estudantes em relação ao apoio à autonomia antes e após um programa de intervenção no ensino dos esportes coletivos nas aulas de educação física.

\section{MÉTODOS}

\section{Amostra}

Participaram do estudo 100 alunos de uma escola estadual de um município da região noroeste do Paraná. Duas turmas de educação física executaram o programa de intervenção (grupo experimental, $G E)$, eram alunos do ensino fundamental de ambos os sexos $\left(6^{\circ}\right.$ ano $=23 ; 7^{\circ}$ ano $\left.=21\right)$. O grupo controle (GC) foi formado por duas turmas de sétimos anos $(n=16 ; n=23)$ e uma turma de sexto ano $(n=17)$.

O programa de intervenção usou um modelo híbrido de ensino (Modelo Desenvolvimentista e Modelo de Educação Desportiva), que tem como objetivo desenvolver os aspectos motores, afetivos e cognitivos (Tabela 1) no ensino dos esportes coletivos. A escolha das turmas foi aleatória, após a apreciação e disponibilidade da escola participante, as aulas do grupo experimental foram dadas durante as aulas de educação física, com a presença e autorização do professor responsável pelas turmas. Foram dadas 45 aulas de 50 minutos, 16 aulas ao futsal, 14 ao basquetebol, 14 ao handebol e uma para avaliação final.

As aulas de educação física foram dadas nos grupos GE1 ( $6^{\circ}$ ano) e GE2 ( $7^{\circ}$ ano), que participaram do programa de intervenção; o grupo controle (GC1 $7^{\circ}$ ano, GC2 $-6^{\circ}$ ano e GC3 $-7^{\circ}$ ano) não participou do programa de intervenção. A maioria dos alunos participantes do estudo $(40,9 \%)$ não pratica esportes fora das aulas de educação física.

O delineamento quase-experimental foi usado para comparar a percepção dos alunos que participaram do programa de intervenção nas aulas de educação física (Grupo Experimental - GE1 e GE2) com o grupo controle. Para efeito de análise dos dados, o grupo 
Tabela 1. Esquematização dos conteúdos (futsal, basquetebol e handebol) para o programa de intervenção pedagógica (grupo experimental).

\begin{tabular}{|c|c|c|c|}
\hline Série & Cognitivo & Motor & Afetivo \\
\hline 60 ano & $\begin{array}{l}\text { - Características dos esportes coletivos; } \\
\text { - Ataques e defesa (direções); } \\
\text { - Igualdade numérica; } \\
\text { - Conceitos iniciais de inferioridade e } \\
\text { superioridade numérica; } \\
\text { - Tomada de decisão tática }\end{array}$ & $\begin{array}{l}\text { - Jogos Desenvolvimentistas de nível II } \\
\text { - Habilidades com bola ( Fundamentos): } \\
\text { a) futsal: drible, passe/recepção e chute; } \\
\text { b) basquetebol: drible, passe e arremesso; } \\
\text { c) handebol: drible, passe e arremesso); } \\
\text { - Exercícios de oposição simplificada: } 1 \times 0,2 \times 1 \text { e 3X2; } \\
\text { - Torneios: Futsal de Trio, Basquete de Dupla e } \\
\text { Handebol de Trio }\end{array}$ & $\begin{array}{l}\text { - Cooperação } \\
\text { - Autoconceito } \\
\text { - Respeito } \\
\text { - Responsabilidade } \\
\text { - Socialização } \\
\text { - Autoestima }\end{array}$ \\
\hline 7ㅇa ano & $\begin{array}{l}\text { - Características dos esportes coletivos; } \\
\text { - Ataque (movimentação ofensiva; papel do } \\
\text { curinga; troca de passes); } \\
\text { - Defesa (introdução à marcação individual); } \\
\text { - Posicionamentos em inferioridade e } \\
\text { superioridade numérica; } \\
\text { - Tomada de decisão tática } \\
\text { - Diferenças e semelhanças entre os } \\
\text { esportes de invasão }\end{array}$ & $\begin{array}{l}\text { - Jogos desenvolvimentistas de Nível II; } \\
\text { - Habilidade com bola (fundamentos: } \\
\text { a) futsal: drible, passe e chute } \\
\text { b) basquetebol: drible, passe e arremesso (bandeja); } \\
\text { c) handebol: drible, passe e arremesso com } \\
\text { progressão); } \\
\text { - Exercícios de oposição simplificada: 1X1, 1X1 } \\
\text { (+1), } 3 \times 2 \text { e } 4 X 3 \text {; } \\
\text { - Exercícios semelhantes ao jogo formal (3X3, 4X4 } \\
\text { e } 5 \times 5 \text { no handebol). } \\
\text { - Torneios: Futsal de Trio, Basquete de Dupla e } \\
\text { Handebol de Trio }\end{array}$ & $\begin{array}{l}\text { - Cooperação } \\
\text { - Autoconceito } \\
\text { - Respeito } \\
\text { - Responsabilidade }\end{array}$ \\
\hline
\end{tabular}

controle, que envolveu alunos que não participaram do programa, foi reorganizado em dois: a) alunos que estudavam na mesma escola do GE (Grupo Controle 1 - GC1), denominado de "Escola 1"; e b) alunos que estudavam em escola em que não foi feito o programa de intervenção (Grupo Controle 2 - GC2 e GC3), denominado de "Escola 2". A aplicação do pré-teste e pós-teste dos grupos (experimental e controle) foi usada para verificar o grau de alteração na percepção dos alunos em relação ao apoio à autonomia fornecida pelos professores. Para tanto, no pré-teste os alunos avaliaram os professores de educação física do ano anterior; no pós-teste suas respostas foram em relação ao professor responsável pelo programa de intervenção. $O$ estudo foi feito somente com alunos que participaram do pré e do pós-teste.

\section{Instrumento}

Foi usado o LCQ, construído por Deci e Ryan (2011) e validado para a língua portuguesa por Costa (2015). 0 questionário tem como objetivo verificar a percepção dos alunos em relação ao apoio à autonomia do professor durante suas aulas. Compreende 15 itens relacionados a situações específicas de aprendizagem, tais como uma disciplina feita por um professor em particular.
As questões estão relacionadas ao apoio à autonomia feita pelo professor durante as aulas. As pontuações são calculadas pela média das respostas. Antes da feitura do cálculo da média das classificações de cada item, primeiro deve-se "inverter" a pontuação do item 13; por exemplo, se for anotado " 3 ", será pontuado " 5 " (exemplo: $1=7,2=6,3=5,4=4,5=3,6=2$ e $7=1$ ). Escores médios mais elevados representam um maior nível de apoio à autonomia dada pelo professor. Após a digitação dos dados no programa Microsoft Excel, usouse uma equação de ponderação adaptada do modelo usado por Lemos, 2007.

A estimativa de consistência interna do questionário foi feita no pré e pós-teste. Os valores do alfa de Cronbach para o questionário indicaram uma boa confiabilidade tanto no pré como no pósteste ( $\alpha=0,81$ e $\alpha=0,84$, respectivamente) de acordo com as recomendações de Nunally (1978).

\section{Coleta de dados}

Inicialmente foi entregue uma carta convite à Chefia do Núcleo Regional de Maringá, que autorizou a feitura do estudo e o contato inicial com as escolas participantes. O programa de intervenção e a coleta de dados se iniciaram após a aprovação do projeto 
pelo Comitê de Ética e Pesquisa com Seres Humanos (parecer n. 236.092). Os dados foram coletados nos horários das aulas de educação física ou em horários agendados.

Foi solicitada autorização dos responsáveis pelos estudantes para participação do estudo através do Termo de Consentimento Livre e Esclarecido.

\section{Análise de dados}

Para análise estatística dos dados, usou-se uma equação de ponderação adaptada do modelo usado por Lemos, 2007, construída no programa Microsoft Excel. O produto final da fórmula matemática do modelo da equação de ponderação é um índice de porcentagem que varia de $-100,00 \%$ a $+100,00 \%$, ou seja, 200 pontos de variabilidade.

Os valores apresentados na escala de variabilidade dessa equação de ponderação do LCQ foram representados da seguinte forma: a) $-100,00 \%$ a $-59,99 \%$ (Índice Muito Baixo); b) $-60,00 \%$ a $-19,99 \%$ (Índice Baixo); c) -20,00\% a +19,99\% (Índice Médio); d) $+20,00 \%$ a +59,99\% (Índice Alto); e) +60,00\% a +100,00\% (Índice Muito Alto). Os índices se referem ao percentual de percepção de apoio à autonomia dos estudantes.

A análise dos dados foi feita por meio da equação de ponderação. Inicialmente fez-se a verificação de normalidade por meio do teste de Shapiro-Wilk, os dados foram considerados não normais. Para caracterização da amostra, foi usada frequência relativa. Como os dados não apresentaram normalidade, foi usada mediana e intervalo interquartil e testes não paramétricos pareados para fazer comparações entre o pré e pós-teste (Wilcoxon). Para comparação entre os grupos no pré e pós-teste, foram usados o teste $U$ de Mann-Whitney e o teste de Kruskal-Wallis, seguidos da comparação múltipla das medianas. O nível de significância adotado foi de $p \leq 0,05$.

\section{RESULTADOS}

Nas comparações entre os resultados da percepção de apoio à autonomia dos alunos participantes do estudo (Tabela 2), verificou-se que não houve diferenças significativas entre o pré e pós-teste. Além disso, nas comparações múltiplas entre os grupos participantes do estudo, os resultados demonstraram que não houve diferenças estatisticamente significativas entre os grupos investigados no pré-teste $(p=0,29)$. Por outro lado, no pós-teste encontraram-se diferenças estatisticamente significativas entre os grupos $(p=0,00)$. No pós-teste, apenas o GC3 obteve índices altos de apoio à autonomia, enquanto as outras turmas obtiveram índices médios. O grupo GE2 apresentou o menor índice em relação a todos os outros grupos, com diferenças estatisticamente significativas entre: GE1 e GE2 ( $p=0,00)$; GE2 e GC1 ( $p=$ $0,02)$; GE2 e GC2 $(p=0,00)$; GE2 e GC3 $(p=0,00)$.

Os alunos do sexo masculino (Tabela 3 ) não apresentaram resultados estatisticamente significativos na comparação entre pré e pós-teste nos índices de apoio à autonomia oferecida pelos professores. Não foram encontradas diferenças estatisticamente significativas entre os grupos GE1/GE2, GC1, e GC2/GC3 no pré-teste $(p=0,62)$ e no pós-teste $(p=0,35)$ no sexo masculino.

Tabela 2. Comparação dos índices de apoio à autonomia (LCQ) na percepção dos alunos do grupo experimental e do grupo controle no pré-teste e pós-teste.

\begin{tabular}{|c|c|c|c|c|c|}
\hline \multirow{2}{*}{ LCQ } & \multicolumn{2}{|c|}{ Pré-teste } & \multicolumn{2}{|c|}{ Pós-teste } & \multirow{2}{*}{ p-valor } \\
\hline & Mediana & (Q1-Q3) & Mediana & (Q1-Q3) & \\
\hline $\begin{array}{l}{[0,1-8] \text { GE1 }(n=23)} \\
\text { Escola } 1\end{array}$ & 22,21 & - 6,68/46,67 & 11,11 & $2,22 / 24,43$ & 0,412 \\
\hline $\begin{array}{l}{[0,1-8] \text { GE2 }(n=21)} \\
\text { Escola } 1\end{array}$ & 00,00 & - 18,88/48,89 & $-13,32^{*}$ & - 33,32/8,89 & 0,058 \\
\hline $\begin{array}{l}{[0,1-8] \text { GC1 }(n=16)} \\
\text { Escola } 1\end{array}$ & 26,66 & 4,99/51,10 & 00,00 & $00,00 / 46,64$ & 0,268 \\
\hline $\begin{array}{l}{[0,1-8] \mathrm{GC2}(\mathrm{n}=17)} \\
\text { Escola } 2\end{array}$ & 35,55 & $10,00 / 55,55$ & 13,33 & $00,00 / 43,32$ & 0,068 \\
\hline $\begin{array}{l}{[0,1-8] \text { GC3 }(n=23)} \\
\text { Escola } 2\end{array}$ & 6,67 & - 4,45/37,77 & 24,43 & $13,31 / 51,09$ & 0,088 \\
\hline & & & & & \\
\hline
\end{tabular}

Obs: (escala de variabilidade): a) -100,00\% a -60,00\% (Índice Muito Baixo); b) -59,99\% a -20,00\% (Índice Baixo); c) -20,00\% a +19,99\% (Índice Médio); d) $+20,00 \%$ a $+59,99 \%$ (Índice Alto); e) $+60,00 \%$ a $+100,00 \%$ (Índice Muito Alto). * Diferença estatisticamente significativa entre GE1 e GE2 $(p=0,00)$; GE2 e GC1 $(p=0,00)$; GE2 e GC2 $(p=0,00)$; GE2 e GC3 $(p=0,00)$. 
Tabela 3. Comparação dos índices de apoio à autonomia (LCQ) na percepção dos alunos do grupo experimental e do grupo controle (sexo masculino) no pré-teste e pós-teste.

\begin{tabular}{|c|c|c|c|c|c|}
\hline \multirow{3}{*}{ LCQ } & \multicolumn{4}{|c|}{ Sexo masculino } & \multirow{3}{*}{ p-valor } \\
\hline & \multicolumn{2}{|c|}{ Pré-teste } & \multicolumn{2}{|c|}{ Pós-teste } & \\
\hline & Mediana & (Q1-Q3) & Mediana & (Q1-Q3) & \\
\hline$[0,1-8]$ GE1 e GE2 $(n=22)$ & \multirow{2}{*}{27,77} & & \multirow{2}{*}{00,00} & & \multirow{2}{*}{0,653} \\
\hline Escola 1 & & $00,00 / 48,33$ & & $-13,89 / 11,66$ & \\
\hline$[0,1-8] \mathrm{GC1}(\mathrm{n}=12)$ & \multirow{2}{*}{17,77} & & \multirow{2}{*}{00,00} & & \multirow{2}{*}{0,248} \\
\hline Escola 1 & & $1,11 / 42,21$ & & $00,00 / 42,20$ & \\
\hline$[0,1-8] \mathrm{GC} 2$ e GC3 $(n=14)$ & \multirow{2}{*}{26,67} & & \multirow{2}{*}{6,67} & & \multirow{2}{*}{0,245} \\
\hline \multirow[t]{2}{*}{ Escola 2} & & $-11,11 / 38,87$ & & $00,00 / 21,65$ & \\
\hline & \multicolumn{2}{|c|}{$p=0,62$} & \multicolumn{2}{|c|}{$p=0,35$} & \\
\hline
\end{tabular}

Obs: (escala de variabilidade): a) -100,00\% a -60,00\% (Índice Muito Baixo); b) -59,99\% a -20,00\% (Índice Baixo); c) -20,00\% a +19,99\% (Índice Médio); d) $+20,00 \% a+59,99 \%$ (Índice Alto); e) $+60,00 \% a+100,00 \%$ (Índice Muito Alto).

Destaca-se, entretanto, que os alunos do sexo masculino não apresentaram maiores índices de percepção ao apoio à autonomia após o programa de intervenção. Ao contrário, em todos os grupos os valores foram inferiores no pós-teste (Tabela 3).

No sexo feminino, as alunas mantiveram os índices de apoio à autonomia em todos os grupos (Tabela 4). No grupo experimental GE1/GE2, as alunas encontravamse com índice médio no pré-teste e mantiveram após o programa de intervenção; nos grupos controle GC1 e CC2/ GC3, as alunas permaneceram no índice alto. Ao comparar os grupos GE1/GE2, GC1 e GC2/GC3 (Tabela 5), foram encontradas diferenças estatisticamente significativas no pós-teste $(p=0,00)$, as alunas do grupo controle (GC1) apresentaram maiores índices de apoio à autonomia em relação ao grupo experimental $(p=0,00)$.

A comparação dos índices de apoio à autonomia nos alunos de $6^{\circ}$ ano (Tabela 6) não indicou diferenças estatisticamente significativas entre o pré e pós-teste no grupo experimental (GE1) e grupo controle (GC2); os índices de apoio à autonomia no pré-teste foram mantidos no pós-teste do grupo experimental (Tabela 5).

Verificaram-se diferenças estatisticamente significativas $(p=0,034)$ nos índices de apoio à autonomia na comparação entre pré e pós-teste dos alunos do GE2 (Tabela 6). O programa de intervenção feito no $7^{\circ}$ ano (GE2) teve um efeito estatisticamente significativo sobre o apoio à autonomia na percepção dos alunos; apesar de terem se mantido no índice médio, os valores foram inferiores no pós-teste. De acordo com a comparação múltipla feita entre os grupos, no pós-teste o grupo controle GC3 apresentou maior percepção de apoio à autonomia (índice alto) do que os alunos do grupo experimental (GE2). Esse último, entretanto, apresentou índices de apoio à autonomia inferior ao GC1.

Tabela 4. Comparação dos índices de apoio à autonomia (LCQ) dos alunos do grupo experimental e do grupo controle (sexo feminino), no pré-teste e pós-teste.

\begin{tabular}{|c|c|c|c|c|c|}
\hline \multirow{3}{*}{ LCQ } & \multicolumn{4}{|c|}{ Sexo feminino } & \multirow{3}{*}{ p-valor } \\
\hline & \multicolumn{2}{|c|}{ Pré-teste } & \multicolumn{2}{|c|}{ Pós-teste } & \\
\hline & Mediana & (Q1-Q3) & Mediana & (Q1-Q3) & \\
\hline $\begin{array}{l}{[0,1-8] \text { GE1 e GE2 }(n=22)} \\
\text { Escola } 1\end{array}$ & $-6,66$ & $-28,89 / 44,33$ & $10,00^{*}$ & $-23,87 / 24,43$ & 0,987 \\
\hline $\begin{array}{l}{[0,1-8] \mathrm{GC1}(\mathrm{n}=04)} \\
\text { Escola } 1\end{array}$ & 43,33 & $13,33 / 71,65$ & $47,76^{*}$ & $9,44 / 69,43$ & 0,952 \\
\hline $\begin{array}{l}{[0,1-8] \mathrm{GC2} \text { e GC3 }(n=26)} \\
\text { Escola } 2\end{array}$ & 20,00 & $4,44 / 44,43$ & 31,11 & $12,20 / 51,64$ & 0,489 \\
\hline & \multicolumn{2}{|c|}{$p=0,09$} & \multicolumn{2}{|c|}{$p=0,00$} & \\
\hline
\end{tabular}

Obs: (escala de variabilidade): a) -100,00\% a -60,00\% (Índice Muito Baixo); b) -59,99\% a -20,00\% (Índice Baixo); c) -20,00\% a +19,99\% (Índice Médio); d) $+20,00 \%$ a +59,99\% (Índice Alto); e) +60,00\% a +100,00\% (Índice Muito Alto). * Diferença estatisticamente significativa entre GE1/ GE2 e GC1 $(p=0,01)$. 
Tabela 5. Comparação dos índices de apoio à autonomia (LCQ) na percepção dos alunos do grupo experimental e do grupo controle (6o ano) no pré-teste e pós-teste

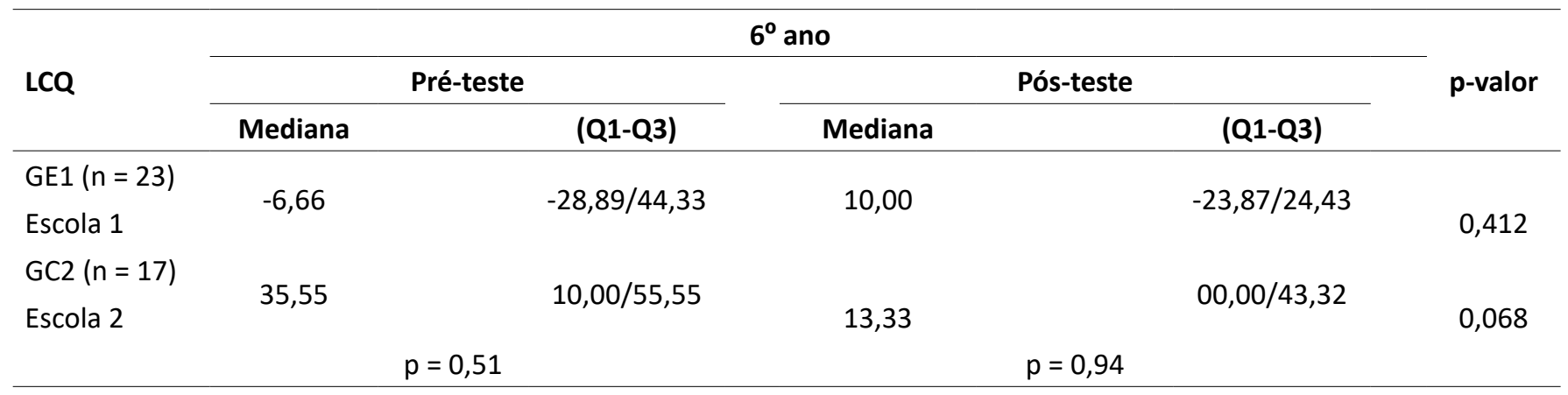

Obs: (escala de variabilidade): a) -100,00\% a -60,00\% (Índice Muito Baixo); b) -59,99\% a -20,00\% (Índice Baixo); c) -20,00\% a +19,99\% (Índice Médio); d) $+20,00 \%$ a +59,99\% (Índice Alto); e) +60,00\% a +100,00\% (Índice Muito Alto).

Tabela 6. Comparação dos índices de apoio à autonomia (LCQ) dos alunos do grupo experimental e do grupo controle (70 ano) no pré-teste e pós-test

\begin{tabular}{|c|c|c|c|c|c|}
\hline \multirow{3}{*}{ LCQ } & \multicolumn{4}{|c|}{$7^{\circ}$ ano } & \multirow{3}{*}{ p-valo } \\
\hline & \multicolumn{2}{|c|}{ Pré-teste } & \multicolumn{2}{|c|}{ Pós-teste } & \\
\hline & Mediana & (Q1-Q3) & Mediana & (Q1-Q3) & \\
\hline $\begin{array}{l}{[0,1-8] \text { GE2 }(n=21)} \\
\text { Escola } 1\end{array}$ & 00,00 & $-18,88 / 48,89$ & $-13,32 a b$ & $-33,32 / 8,89$ & $0,034 *$ \\
\hline $\begin{array}{l}{[0,1-8] \mathrm{GC1}(\mathrm{n}=16)} \\
\text { Escola } 1\end{array}$ & 26,66 & $-4,99 / 51,10$ & $00,00 a$ & $00,00 / 46,64$ & 0,268 \\
\hline $\begin{array}{l}{[0,1-8] \text { GC3 }(n=23)} \\
\text { Escola } 2\end{array}$ & 6,67 & $-4,45 / 37,77$ & $24,43 b$ & $13,31 / 51,09$ & 0,088 \\
\hline & & & & & \\
\hline
\end{tabular}

Obs: (escala de variabilidade): a) -100,00\% a -60,00\% (Índice Muito Baixo); b) -59,99\% a -20,00\% (Índice Baixo); c) -20,00\% a +19,99\% (Índice Médio); $d)+20,00 \%$ a $+59,99 \%$ (Índice Alto); e) $+60,00 \% a+100,00 \%$ (Índice Muito Alto). * Diferença significativa entre a = GE2 e GC1 ( $p=$ $0,01)$; diferença significativa entre $b=$ GE2 e GC3 $(p=0,00)$.

\section{DISCUSSÃO}

O presente trabalho, ao fazer um programa de intervenção, procurou analisar a percepção dos alunos em relação aos seus professores no que se refere ao apoio à autonomia durante as aulas de educação física. A TAD evidencia que o apoio à autonomia pode contribuir para a melhoria da motivação e percepção de competência dos indivíduos. No ambiente escolar, os professores poderiam colaborar em uma formação mais adequada dos estudantes se soubessem fornecer apoio à autonomia de seus alunos. No âmbito da educação física, estudos têm demonstrado que os alunos mais motivados nas aulas participam efetivamente das atividades e acreditam em sua importância (Carreiro da Costa et al., 1997; Cervelló, 1999).

A principal conclusão que emerge deste estudo se refere ao fato de que o programa de intervenção não foi suficiente para apresentar alterações estatisticamente significativas na percepção dos alunos em relação ao apoio à autonomia oferecido pelos professores, independentemente dos grupos investigados e do sexo dos alunos. No $7^{\circ}$ ano (grupo GE2) os valores encontrados foram inferiores no pós-teste, apresentaram índices médios de apoio à autonomia. Dessa forma, conforme se avançou no nível de escolaridade, os resultados demonstraram um menor índice de apoio à autonomia dos estudantes, em que os alunos do $7^{\circ}$ ano do grupo experimental (GE2) tiveram dificuldade de se envolver efetivamente nas atividades desenvolvidas no programa de intervenção, principalmente nas primeiras aulas, o que pode decorrer das suas experiências anteriores (Tabela 4). Porém, é necessário ressaltar que esses alunos apresentaram índice médio no pré-teste e mantiveram após o programa de intervenção, ou seja, as alterações não foram estatisticamente significativas com esse tempo de intervenção.

Um dos objetivos das aulas dadas durante o programa de intervenção foi buscar intensificar o apoio à autonomia dos estudantes, pois os estudos sobre 
motivação na área educacional têm mostrado que os professores que promovem o apoio à autonomia têm alunos que passam a regular internamente seu comportamento, o que aumenta sua autoestima, o estabelecimento de metas e, consequentemente, a sua motivação intrínseca (Deci et al., 1981; Grolnick e Ryan, 1987; Deci et al., 1991). Entretanto, ao analisar o apoio à autonomia em função do sexo, verificouse que a programa de intervenção não alterou a percepção dos alunos tanto para o sexo masculino (Tabela 2) quanto feminino (Tabela 3). Os índices inferiores no pós-teste para o sexo masculino podem refletir a desmotivação dos alunos durante as aulas. A literatura aponta que a falta de apoio à autonomia está fortemente correlacionada com a desmotivação dos estudantes (Mazyari et al., 2012; Shen et al., 2010).

As diferenças nas percepções do apoio à autonomia entre as turmas de $6^{\circ}$ e $7^{\circ}$ ano podem se confirmar em atividades feitas durante o programa de intervenção. Enquanto os estudantes do $6^{\circ}$ ano entregaram suas tarefas de casa no prazo determinado, os da $7^{\circ}$ série somente as entregavam com pressões externas, como as notas na disciplina, o que pode ter influenciado em sua percepção diminuída após o programa de intervenção (Tabela 4). De acordo com a TAD, quando os alunos fazem atividades que consideram pouco interessantes, como as tarefas, são regidos por um lócus de causalidade externa (Deci e Ryan, 2000). A teoria da causalidade das orientações, uma das miniteorias da TAD, descreve e analisa três tipos de orientações de causalidade: a orientação de autonomia, em que as pessoas agem por interesse e valorização de si mesmas; a orientação de controle, em que o foco está em recompensas, ganhos e aprovação, e a orientação impessoal caracterizada pela falta de motivação.

As estudantes do sexo feminino também apresentaram características muito diferenciadas em relação ao sexo masculino nos aspectos relacionados aos conteúdos do programa de intervenção (Tabela 5). A maioria das alunas parece compreender mais rapidamente a importância de sua execução, questionando, participando efetivamente e entregando as tarefas de casa no prazo determinado. Dessa forma, para a TAD, os professores deveriam demonstrar a importância de cada conteúdo e atividades desenvolvidas, de modo que os estudantes compreendam a sua necessidade em suas vidas. Durante as atividades esportivas desenvolvidas em meio ao programa de intervenção, o professor identificou os benefícios psicológicos da prática esportiva, com atividades teóricas e práticas (trabalhos, testes, grupos de trabalho) sobre as características dos esportes coletivos que lhe forneciam feedback do trabalho feito, estimulando o bem-estar psicológico advindo da autonomia dos sujeitos (Deci e Ryan, 2002). A melhoria da compreensão dos benefícios das aulas no fim do programa de intervenção, quando os alunos participavam nos eventos no fim de cada modalidade (torneios), levou o índice de apoio à autonomia a apresentar índices médios a altos, apesar dos índices não terem sido maiores no pós-teste.

A maior percepção de apoio à autonomia no grupo controle da escola 2 em comparação com alunos do grupo experimental e do grupo controle da escola 1 demonstrou que, na percepção dos alunos, os professores da escola 2 parecem desenvolver maior apoioà autonomia (Tabela 5). Estudos demonstram que os alunos com maior motivação são aqueles que no futuro mais praticam atividade física e os mais propensos à adesão aos hábitos esportivos durante o lazer (Granero-Gallegos et al., 2014), o que demonstra a preocupação com o estímulo aos alunos nas aulas de educação física.

$\mathrm{Na}$ atualidade, os conteúdos a serem aprendidos no ambiente escolar devem fazer sentido para os alunos e isso se inicia no trabalho docente. Dessa forma, o apoio à autonomia e o controle da regulação decorre do comportamento dos envolvidos no processo de ensino e aprendizagem (Ryan e Deci, 2000).

\section{CONCLUSÃO}

Apesar de os alunos não terem percebido alterações no apoio à autonomia oferecido pelos professores após o programa de intervenção, os índices encontrados apontaram uma manutenção na percepção dos alunos, mesmo nos alunos de $6^{\circ}$ ano que vinham de outro ambiente escolar. Além disso, os alunos em geral não apresentaram índices baixos e muito baixos, ou seja, na percepção dos estudantes os professores ofereceram apoio à autonomia (índice médio e alto) antes e após o programa de intervenção.

Assim, faz-se necessária a compreensão dos diferentes aspectos psicológicos inerentes ao contexto educacional, pois esse representa o primeiro momento de socialização dos alunos, exerce grande impacto na vida desses sujeitos, no seu comportamento e no contexto da aprendizagem.

O estudo se baseou nas respostas dos alunos, mas não foram analisados nessa investigação dados objetivos da prática pedagógica dos professores de cada turma investigada para verificar se os professores forneceram apoio à autonomia durante suas aulas. Uma das limitações do estudo está relacionada aos fatores sociocontextuais, como, por exemplo, o tempo de experiência no magistério, o sexo, a idade, as interações com a direção da escola, que não foram correlacionadas neste estudo e podem interferir no apoio à autonomia. 
Em estudos futuros, sugere-se que indicadores objetivos, como observação sistemática das aulas e entrevistas com os professores, possam ser considerados.

\section{CONFLITOS DE INTERESSE}

Os autores declaram não haver conflitos de interesse.

\section{REFERÊNCIAS}

Ames C. Classrooms: Goals, structures, and student motivation, Journal of Educational Psychology.1992;84:261-71.

Assor A, Kaplan H, Kanat-Maymon Y, Roth G. Directly controlling teacher behaviors as predictors of poor motivation and engage- ment in girls and boys: The role of anger and anxiety. Learning and Instruction. 2005;15:397-413.

Cervelló EM. Variables psicológicas relacionadas con la elección de tareas con diferente nivel de dificultad: implicaciones para el desarrollo de programas de entrenamiento psicológico motiva- cional en deporte. Motricidad 1999;5:35-52.

Costa LCA. Influência de um programa de ensino de esportes coletivos de invasão na motivação e desempenho motor de esco- lares do ensino fundamental. Maringá: Universidade Estadual de Maringá/Universidade Estadual de Londrina; 2015, Tese de doutorado.

Cox AE, Smith AL, Williams L. Change in physical education moti- vation and physical activity behavior during middle school. The Journal of Adolescent Health 2008;43:506-13.

Deci EL, Ryan RM. The "what" and "why" of goal pursuits: Human needs and the self-determination of behavior. Psychological Inquiry 2000;11:227-68.
Deci EL, Schwartz AJ, Sheinman L, Ryan RM. An instrument to assess adults' orientations toward control versus autonomy with children: Reflections on intrinsic motivation and perceived com- petence. Journal of Educational Psychology 1981;73:642-50.

Deci EL, Ryan RM. Intrinsic motivation and self-determination in human behavior. New York: Plenum; 1985.

Grolnick WS, Ryan RM. Autonomy in children's learning: an experimental and individual differences investigation. Journal of Personality and Social Psychology 1987;52:890-8.

Lemos CAF. Qualidade de vida na carreira profissional de professores de educação física do magistério público estadual/RS. 115 f. Dissertação (Mestrado em Educação Física) -Centro de Desportos, Universidade Federal de Santa Catarina, Florianópolis, 2007. Disponível em: http://tede.ufsc.br/teses/ PGEF0152.pdf. Acesso em: 23 jul 2013.

Granero-Gallegos A, Baena-Extremera A, Sanchez-Fuentes JA, Martinez-Molina M. Perfiles motivacionales de apoyo a la autonomía, autodeterminación, satisfacción, importancia de la educación física e intención de práctica física en tiempo libre. Cuadernos de Psicología del Deporte 2014;14(2): 59-70.

Ryan RM, Deci EL. Self-determination theory and the facilitation of intrinsic motivation, social development, and well-being. Ame- rican Psychologist 2000;55:68-78.

Ryan RM, Connell JP. Perceived locus of causality and internalization. Journal of Personality and Social Psychology 1989;5:749-61.

Shen B, Sun LIW, Rukavina HPB. The Influence of Inadequate Teacher-to-Student Social Support on Amotivation of Physical Education Students. Journal of Teaching in Physical Education 2010;29(4):417-32. 